\title{
O parque verde urbano: características do uso através de observação sistemática
}

\author{
The green urban park: characteristics of use through systematic observation
}

Fernanda Berguerand Xavier ${ }^{[0]}$, Juliana Felipe ${ }^{[0]}$, Alba Regina Azevedo Arana ${ }^{[0]}$

[a] Universidade do Oeste Paulista (UNOESTE ), Presidente Prudente, SP, Brasil

\section{Resumo}

Entre inúmeros benefícios, parques urbanos promovem oportunidades para a prática de atividades físicas (AF). Em Presidente Prudente - SP, o Parque do Povo é o principal espaço verde público de lazer da cidade. Este artigo objetiva verificar a relação entre parques e AF, e discutir seu potencial em estratégias ambientais de promoção de saúde. 0 nível de AF e características do uso do parque foram coletados através do System for Observing Play and Recreation in Communities (SOPARC), instrumento que se baseia em observações sistemáticas. A coleta de dados realizou-se em 2015, e 19.105 indivíduos foram observados. 0 período noturno foi o mais frequentado (53\%), a AF moderada predominou (54\%), e a sedentária aumentou aos finais de semana. Adultos representaram 59\% da amostra, e os homens 65\%. Os resultados mostram relação positiva entre o parque e a prática de atividades físicas, e que determinados períodos do dia e da semana, bem como outras preferências dos usuários devem ser observadas para o aumento da eficácia de programas voltados à promoção da saúde em ambientes urbanos.

Palavras-chave: Parques urbanos. Promoção de saúde. Áreas verdes. Gestão urbana.

\section{Abstract}

Among numerous benefits, urban parks promote opportunities for the practice of physical activities (PA). In Presidente Prudente - SP, the Parque do Povo is the main public space for leisure in the city. This article aims to verify the relationship between parks and $P A$, and discuss its potential in health promotion environmental strategies. The level of PA and characteristics of its use have been collected through the System for Observing Play and Recreation in Communities (SOPARC), tool that is based on systematic observations. The data collection was carried out in 2015 and 19,105 individuals have been observed. The night period was the more frequented overall (53\%), the moderated PA was predominant (54\%), and sedentary activities have increased on weekends.

FBX é mestre em Meio Ambiente pela Universidade do Oeste Paulista, e-mail: fernandaberguerand@hotmail.com JF é mestre em Meio Ambiente pela Universidade do Oeste Paulista, e-mail: jfedfisica@gmail.com ARAA é doutora em Geografia pela Universidade de São Paulo e coordenadora do Mestrado em Meio Ambiente e Desenvolvimento Regional, e-mail: alba@unoeste.br 
The adults represented 59\% of the sample, and men $65 \%$. The results shows a positive relationship between the park and practice of physical activities and that certain periods of the day and week and other preferences of the users have to be observed for efficient programs of health promotion in urban environments.

Keywords: Urban parks. Health promotion. Green area. Urban management.

\section{Introdução}

Um espaço urbano de qualidade é um fator decisivo para a melhor qualidade de vida e saúde de uma população. Uma série de estudos tem mostrado que a vida em ambientes mais naturais influencia positivamente a auto percepção de saúde das pessoas e leva a um menor risco de mortalidade (Maas et al., 2008). Um mecanismo sugerido para isso é que a oferta de espaços verdes urbanos promova a atividade física (AF), reduza a obesidade e aumente a saúde respiratória e cardiovascular (Branas etal., 2011). Díaz (2005) considera que a vegetação torna o ambiente muito mais atrativo, dá a sensação de refúgio do dia-a-dia, alivia o stress e estimula as pessoas à prática de atividades físicas.

O sedentarismo é apontado como problema de saúde púbica no Brasil. Dados do Instituto Brasileiro de Geografia Estatística (IBGE) em pesquisa divulgada em dezembro de 2014 apontam que $46 \%$ da população com idade superior a 18 anos é sedentária, não atingindo a recomendação de 150 minutos de AF por semana, seja nos momentos de lazer, no trabalho ou nos deslocamentos até o trabalho (IBGE, 2014). Ainda segundo a pesquisa, o percentual de pessoas que praticam atividades físicas no lazer diminui de acordo com a faixa etária. De 18 a 24 anos, a proporção é de 35\%. Essas taxas vão caindo para $25 \%$ (de 25 a 39 anos), 18\% (de 40 a 59 anos) e 13\% (de 60 anos ou mais).

As grandes concentrações urbanas, a redução dos espaços livres, assim como a revolução tecnológica criaram cenários que predispõem ao estilo de vida inativo (Collet et al., 2012). Ainda que constitua apenas um único fator, parques urbanos são espaços públicos comuns que podem desempenhar um papel significativo no aumento dos níveis de atividade física da população. Eles fornecem locais propícios para que as pessoas caminhem ou corram, muitas vezes possuem instalações específicas para esportes, exercícios e outras atividades vigorosas, oferecendo a oportunidade de incluir atividades físicas nos momentos de lazer de forma gratuita.

0 desporto praticado ao ar livre é baseado numa concepção mais aberta e multidimensional. Deixa de ser reservado só para alguns e passa a ser uma atividade de várias opções, tornando-se assim um desporto para todos. É necessário oferecer às pessoas condições para a prática esportiva e ocupação do tempo livre de uma forma autônoma, livre de horários. Os parques urbanos possibilitam essa prática, abrangendo todas as faixas etárias e sociais, e dão condições necessárias para um desenvolvimento individual (Nunes, 1999).

Desafios logísticos e até mesmo custos financeiros dificultam a pesquisa que envolve a relação entre parques urbanos e a prática de $\mathrm{AF}$, que ainda é reduzida. Muitas são as barreiras encontradas na tentativa de traçar um perfil dos frequentadores, bem como enumerar as características desses ambientes que atraem pessoas para seu uso ativo. Essa dificuldade pode estar relacionada à diversidade de metodologias utilizadas, à indefinição de qualidade dos espaços, às medidas e definições de espaço verde, às diferentes populações e localizações geográficas abordadas, bem como aos instrumentos de autor-relato de atividade física que nem sempre expõem a realidade.

No Brasil, ainda são raras as publicações que dissertam sobre a importância dos parques urbanos para a AF (Reis, 2001; Collet et al., 2012; Silva et al., 2009; Cassou, 2009), sendo essas concentradas na região sul do país em sua maioria. A escassez de informações quanto à forma de utilização e os fatores que podem facilitar ou impedir a utilização dos parques de maneira ativa, traz à tona a necessidade de compreensão dos fatores que possam intervir na ocupação de parques urbanos enquanto espaço de lazer (Collet et al., 2012).

Desta forma, este artigo tem como objetivo verificar a relação entre parques e a atividade física, discutir seu potencial em estratégias ambientais de promoção de saúde enfocando as características do uso do Parque do Povo. Este artigo, portanto, traz as seguintes reflexões: em que extensão estas áreas promovem a prática de atividade física minimizando o sedentarismo? Quais os determinantes para o uso de espaços verdes urbanos, em especial o Parque do Povo em Presidente Prudente? Qual o principal perfil destes frequentadores? 


\section{Determinantes para o uso de parques verdes urbanos}

Os fatores utilizados para medir o uso dos parques variam em termos de período, tempo de atividade, tipos estudados, entre outros. Isso produz estimativas igualmente variadas de prevalência de seu uso. Mensurar o nível de AF realizada nestes locais e a relevância dos mesmos na estratégia de promoção de saúde através de uma população mais ativa torna-se um desafio multidisciplinar.

Nos Estados Unidos e na Austrália as atividades passivas realizadas nos parques superam a prática de AF regular (Giles-Corti et al., 2005). Na Austrália, por exemplo, a prevalência do uso adulto de parques para a AF nas duas semanas anteriores ao estudo variou de $13 \%$ a $17 \%$ do total de frequência (Giles-Corti et al., 2005). Já no Brasil, um estudo realizado por Reis (2001) na cidade de Curitiba constatou que a maioria da população presente no Jardim Botânico encontra-se fisicamente ativa, e, ainda, que $61,85 \%$ desses indivíduos atingem as recomendações para a prática de AF. À proporção disso, Brownson et al. (2000) constatou que em 50 estados americanos somente $38 \%$ da população analisada atingiu as mesmas recomendações.

Inúmeros fatores observáveis podem influenciar o uso dos parques urbanos. Uma revisão da literatura por Broomhall (2006) incluiu a esses: as características do próprio parque - qualidade e quantidade do espaço disponível; o acesso a instalações locais - quadras e centros de lazer; a relação entre atributos do parque e necessidades dos usuários locais; a manutenção do parque, e ainda os fatores de segurança percebida.

Em sua proposta conceitual, Bedimo-Rung et al. (2005) propõem um modelo para guiar pensamentos e sugerir hipóteses, e define alguns atributos que podem ser utilizados como padrão para futuras pesquisas. Nesta classificação, a discussão centra-se sobre as características ambientais do parque que poderiam estar relacionados à $\mathrm{AF}$, sendo essas: as características do ambiente, condições, facilidade ou dificuldade de acesso, estética do local, segurança percebida e políticas voltadas a esse objetivo. De outra maneira, Tinsley et al. (2002) atribui o uso a fatores como: proximidade, acessibilidade, aspectos estéticos como a presença de árvores, água (lago) e existência de pássaros, manutenção do parque, tamanho e a disponibilidade de facilidades como calçadas ou percursos para pedestres (caminhada).
Em meio à diversidade de variáveis, existe certo consenso encontrado em um número significativo de pesquisas ao se tratar da relação positiva entre o fácil acesso e/ou proximidade a parques urbanos e o aumento do nível de AF nesses locais (Reis, 2001; Giles-Corti et al., 2005; Cohen et al., 2007; Kaczynski \& Henderson, 2007; Kaczynski et al., 2008; Sugiyama etal., 2010; Coombes et al., 2010; Veitch et al., 2012). Em um estudo recente realizado nos Estados Unidos, participantes relataram-se propensos a visitar estes locais desde que não seja necessário atravessar ou viajar em estrada de tráfego intenso (Veitch et al., 2014).

A despeito disso, ao explorar a disponibilidade de acesso a parques urbanos e sua relação com a prática de atividades físicas, muitos autores não encontraram relação alguma em Norwich, na Inglaterra (Hillsdon et al., 2006), nos parques da Georgia e Missouri nos Estados Unidos (Hoehner et al., 2005), em estudo nacional realizado na Nova Zelândia (Witten et al., 2008) e também na Escócia (Ord et al., 2013). Esses autores partem da premissa que estes espaços são influentes na saúde das populações estudadas, mas desconsideram que a prática de AF seja o mecanismo que explique essa associação. 0 papel desses locais na saúde mental desses indivíduos poderia ser a possível explicação para essa associação.

Ainda, ao analisar a relação entre vegetação e prática de atividades físicas, Maas et al. (2008) através de estudo realizado na Nova Zelândia, concluem que a quantidade de espaço verde no ambiente de vida é dificilmente relacionada com o nível de AF. Para os autores, a quantidade de AF realizada em ambientes de vida mais ecológicos não explica a relação entre o espaço verde e saúde. Esses resultados adversos dificultam a elucidação da temática em questão.

Bargos (2010) admite que as áreas verdes tendam a assumir diferentes papéis na sociedade e suas funções devem estar inter-relacionadas no ambiente urbano, de acordo com o tipo de uso a que se destinam (Figura 1).

Apesar disso, a estética e características do parque também têm sido positivamente associadas à visitação do mesmo para a manutenção da vida ativa (Giles-Corti et al., 2005; Reis et al., 2009). Ao revisar 18 estudos, Owen et al. (2004) concluiu que fatores estéticos e a presença de colinas estavam diretamente associados a atividades físicas auto percebidas, apesar de nenhum desses estudos reportar qual o tipo de $\mathrm{AF}$ que ocorreu nesses locais. Na cidade de Curitiba, a beleza 


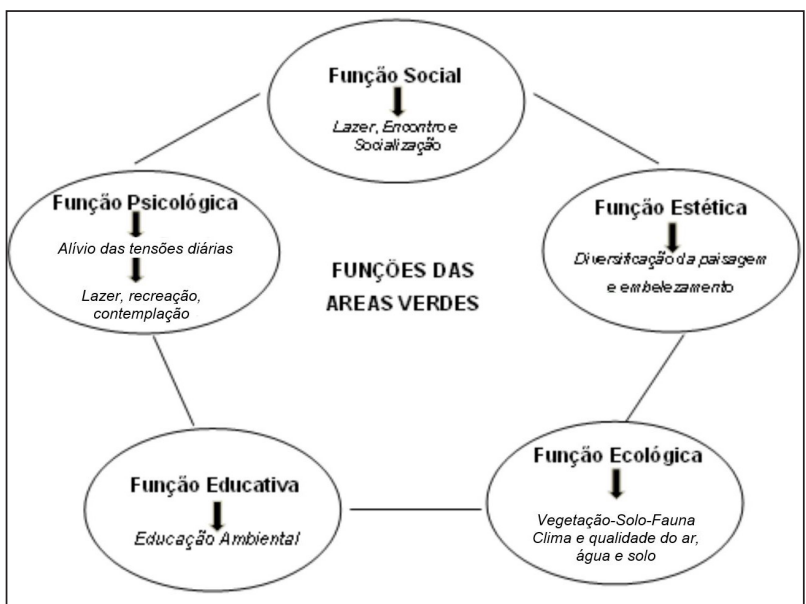

Figura 1 - Funções das Áreas Verdes Urbanas

Fonte: Bargos, 2010.

geográfica do parque e as pistas de caminhada/corrida foram atribuídas como incentivadoras da prática de AF por mais de $94 \%$ dos entrevistados (Reis, 2001).

O tamanho dos parques foi considerado por Coombes et al. (2010) uma característica essencial na busca de níveis de AF e diminuição do sobrepeso. No que concerne a isso, Cohen et al. (2007) concluíram que recursos, mais do que o tamanho do parque, atraem pessoas para a prática de atividades físicas, incluindo acessibilidade, disponibilidade e qualidade das facilidades. Kaczynski et al. (2008), por sua vez, classifica a presença de trilhas - pavimentadas, não pavimentadas e arborizadas - como os fatores determinantes da prática em questão, e desconsidera a influência da estética e segurança do parque nos 33 parques analisados no Canadá.

No Brasil, em estudo feito em Florianópolis por Collet et al. (2012) observou-se que os equipamentos disponíveis no parque não se apresentaram como fatores relevantes, contrariando os achados de Cohen et al. (2007). A mesma escala de auto percepção do ambiente utilizada pelos autores foi posteriormente utilizada por Silva et al. (2009) em Curitiba. Curiosamente, o indicador "equipamentos disponíveis" se apresentou como fator fortemente estimulador para a prática de atividades físicas. Observou-se que no segundo estudo, a população que reportou esse fator como estimulante advinha de famílias com baixo nível sócio econômico. Isso pode indicar que quanto mais baixa a renda do indivíduo, maior a tendência em perceber esse fator como estimulador. Uma possível explicação seria que pessoas de baixa renda têm poucas oportunidades
Tabela 1 - Motivacõoes para a prática de atividades físicas em 2013 no Brasil

\begin{tabular}{lc}
\hline \multicolumn{1}{c}{ Variável } & (\%) \\
\hline Atividade Física & 36 \\
Para minha qualidade de vida e bem estar & 29 \\
Para melhoria no desempenho físico & 12 \\
Para relaxar no meu tempo livre & 9 \\
Para me relacionar com os meus amigos e/ou fazer novas amizades & 5 \\
Para melhoria na harmonia corporal (corpo/mente) & 4 \\
Para competir com outros e comigo & 5 \\
Outros (indicação médica, prêmios, bolsas) & \\
\hline
\end{tabular}

Fonte: Brasil, 2015.

de praticar atividades físicas em locais particulares (clubes e academias) e valorizam ambientes públicos com equipamentos disponíveis gratuitamente (Bedimo-Rung et al., 2005; Silva et al., 2009).

Quanto às motivações para a prática de $\mathrm{AF}$, a pesquisa mostrou que a qualidade de vida e bem-estar são os maiores responsáveis pela busca de uma vida ativa (Tabela 1).

Em relação ao meio ambiente em que ocorre apenas um terço da população brasileira declarou frequentar locais pagos para a prática de AF. Quanto ao fator tecnológico e arquitetônico do ambiente no parque, os resultados encontrados em Florianópolis (Collet et al., 2012) também diferiram quando comparados aos de Curitiba (Silva et al., 2009). Enquanto Silva et al. (2009) observaram que em Curitiba a pista de caminhada no parque, estacionamento e beleza arquitetônica das estruturas construídas foram fatores estimulantes para a população analisada, Collet et al. (2012) concluíram que no parque de Florianópolis podem ser considerados estímulos positivos somente a estética arquitetônica das estruturas construídas e a pista de caminhada e corrida, sendo considerado indiferente o estacionamento no parque.

Outro estudo realizado em Curitiba associou qualidade ambiental à frequência no uso de parques e praças (Cassou, 2009). A maioria dos usuários destes espaços estudados indicou ser a beleza e presença de estacionamento os fatores mais significativos. Já os frequentadores das praças consideram imprescindíveis a proximidade de casa, estrutura, equipamentos e segurança percebida. 
A utilização dos parques não depende apenas de atributos que o local oferece, mas também se relaciona com preferências individuais como idade, hábitos de exercício e raça/etnia (Cohen et al., 2007). Broomhall (2006) destaca as características dos potenciais utilizadores como de extrema importância para sua adesão à $\mathrm{AF}$ nesses ambientes, enumerando-as por: status socioeconômico, idade, gênero, etnia, e fatores psicológicos como auto eficácia e barreiras individuais que influenciam as preferências pessoais.

Em proposta conceitual, Bedimo-Rung et al. (2005) enumeram também as razões pessoais mais comuns para não se envolver em atividades nos parques, que incluem a falta de tempo, dinheiro, saúde pessoal, informação, transporte e acesso, preocupações de segurança e também a falta de companheiros de lazer.

Os resultados encontrados até o presente momento, apesar de variados, apontam alguns perfis e preferências em questão. Bedimo-Rung et al. (2005) constataram que os adultos mais velhos, mulheres e as famílias de renda mais baixa são menos propensos a frequentarem os parques públicos para a prática de AF. A despeito disso, Reis (2001) aponta resultados diferentes no Jardim Botânico em Curitiba: igualdade na distribuição entre homens e mulheres e predomínio de adultos acima de 35 anos. Posteriormente, também em Curitiba, Cassou (2009) constatou que quanto maior a qualidade ambiental dos parques e praças, maior é a utilização destes por mulheres, idosos e pessoas escolarizadas.

Hillsdon et al. (2006), assim como Bedimo-Rung et al. (2005) também encontraram na Inglaterra uma diferença significante de gêneros fisicamente ativos nos parques de Norwich: os homens são duas vezes mais ativos que as mulheres. Cohen et al. (2007), que também observaram essa diferença, chamou a atenção para a ausência de mulheres em atividades vigorosas. Os autores observaram a presença de mulheres em atividades sedentárias ou em locais que estivessem observando crianças, sendo pouco presente em campos, quadras e locais de jogos competitivos. Chama a atenção o fato de essa diferença significativa de gênero ativo não ter sido encontrada nos parques analisados em Florianópolis e Curitiba por Reis (2001) e Cassou (2009), o que sugere que as mulheres brasileiras podem estar mais conscientes quanto a necessidade de exercícios físicos no dia a dia.

Os fatores "valores e atitudes" foram considerados estimulantes em parques de Florianópolis, uma vez que os indicadores de comportamento frequentemente observados dos usuários, apoio/incentivo de amigos e imagem atribuída pela comunidade, foram declarados positivamente associados à realização de atividades físicas no local (Collet et al., 2012). Os resultados encontrados confirmam as evidências de outros estudos que indicam que há associação positiva entre atividades físicas em parques e aspectos como o apoio e estímulo vindo de familiares e amigos, além do contato com demais pessoas que também realizam atividades físicas (Collet et al., 2012), sugerindo que observar pessoas praticando atividades físicas pode constituir um fator intrinsecamente estimulante para a auto percepção de saúde e busca por uma vida mais ativa.

0 fator econômico da população no local em que o parque se insere também demonstra influência na questão estudada. 0 nível social do bairro determina muitas vezes a acessibilidade aos parques, combinando fator individual com determinante físico. Veitch et al. (2014) constataram que adolescentes que vivem em bairros de classe social mais baixa costumam ter que se deslocar duas vezes mais do que os adolescentes de classe alta para chegar ao parque mais próximo, sugerindo que essa disparidade dificulte a frequência dos primeiros. Por outro lado, Bedimo-Rung et al. (2005) e Silva et al. (2009) defendem o fato que pessoas de baixa renda atribuem mais valor aos locais públicos que oferecem oportunidades de lazer e $\mathrm{AF}$, o que explicaria o importante papel desses locais no Brasil.

Sendo assim, o uso dos parques para a prática de atividades físicas atrela-se à subjetividade. Muitos são os determinantes físicos e individuais que incutem motivações intrínsecas ao ser humano na busca de saúde através da utilização desses espaços. A diversidade de população e localizações geográficas apresentados nesse estudo sugere que a própria distribuição desigual de espaços públicos abertos em todas as cidades e entre os países podem explicar as variações interculturais e sócio econômicas abordadas.

\section{Parque do Povo: o objeto de estudo}

Presidente Prudente é um município brasileiro localizado na Alta Sorocabana, no extremo oeste do estado de São Paulo, a $22^{\circ} 07^{\prime} 04^{\prime \prime}$ de latitude sul e $51^{\circ} 22^{\prime} 57^{\prime \prime}$ de longitude oeste. Junto às divisas do Paraná e Mato Grosso do Sul, pertence à mesorregião e microrregião homônimas, distante 558 quilômetros da capital estadual, São Paulo. Ocupa uma área de $562,107 \mathrm{~km}^{2}$, sendo $16,56 \mathrm{~km}^{2}$ de área urbana. Sua população no ano de 2014 era de 220599 habitantes, 
sendo $36^{\circ}$ mais populoso de São Paulo e primeiro de sua microrregião (Presidente Prudente, 2014).

Atualmente a cidade se tornou um dos principais polos industriais, culturais e de serviços do oeste de São Paulo, tanto é que passou a ser conhecida como a "Capital do Oeste Paulista". Segundo o censo de 2000, 48\% da população eram homens (91 797 habitantes) e 52\% (97389 habitantes) mulheres. Cerca de, 97\% (185 229 habitantes) vivia na zona urbana e 2\% (3 957 habitantes) na zona rural (Presidente Prudente, 2014). Seu clima é considerado como Tropical Úmido Seco, o qual representa verão úmido e inverno seco. As variações de temperatura evidenciam um ciclo sazonal que estabelece duas estações bem definidas. Nas áreas inseridas na malha urbana, as temperaturas são mais elevadas e a umidade relativa é mais baixa (INMET, 2017; IPMet, 2005; Amorim, 1993).

o Parque do Povo, localizado na porção sudoeste da cidade, é um espaço verde urbano que possui forma uma faixa linear, de cerca de $3 \mathrm{~km}$ de extensão constituída por grandes áreas de gramado, além de áreas de múltiplo uso, que incluem calçadas, pistas, ginásios e quadras para práticas desportivas (Figura 2).

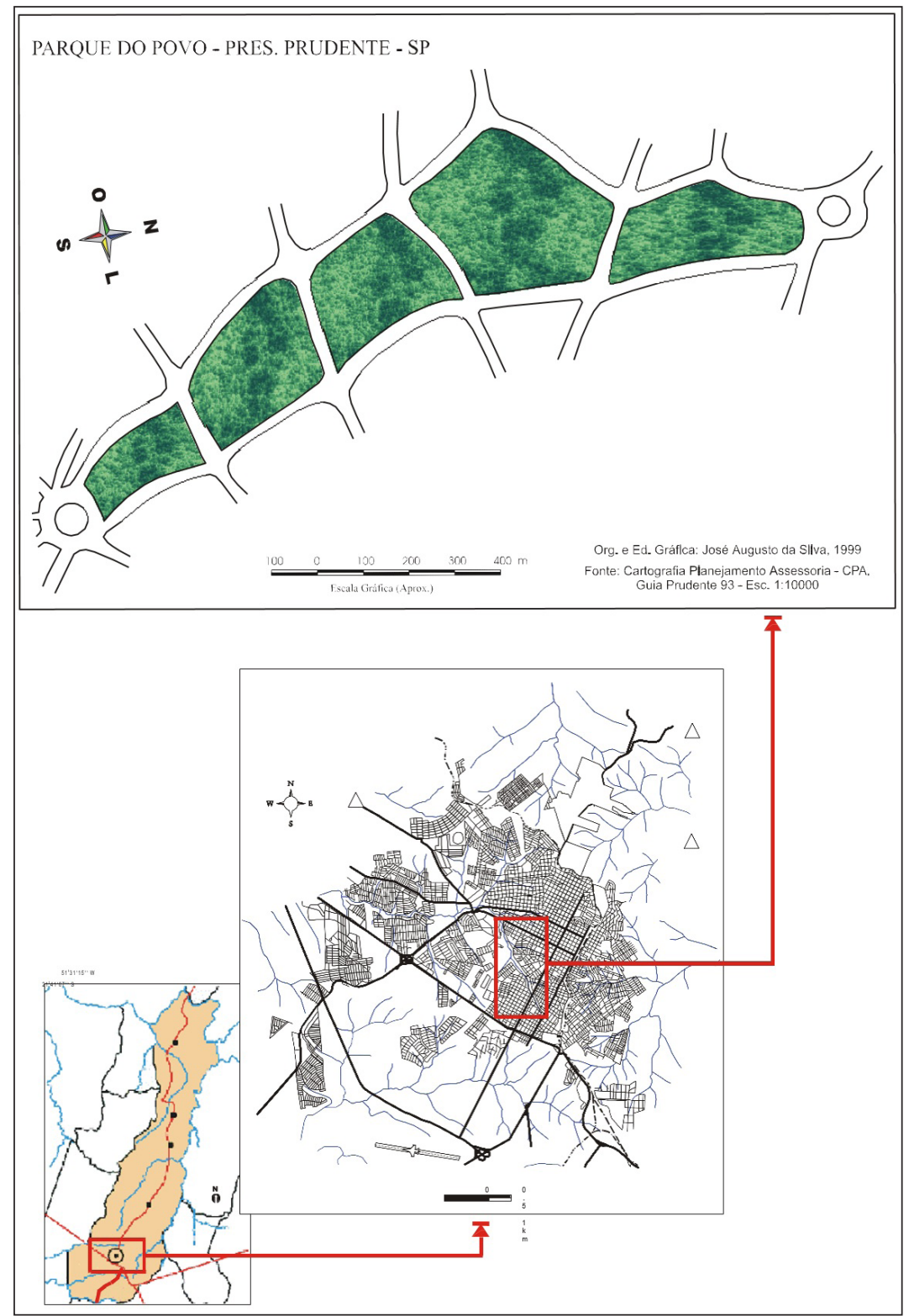

Figura 2 - 0 Parque do Povo e parte da malha urbana de Presidente Prudente - SP Fonte: Silva, 2001. 
Inicialmente projetado para recuperar uma área de fundo de vale, o parque passou por muitas dificuldades no decorrer de sua implantação. Fatores naturais e financeiros, por vezes, inviabilizaram e paralisaram o desenvolvimento da área. Como decorrência dos investimentos realizados pelo Poder Público, tanto a área como o seu entorno vieram a sofrer transformações bastante significativas na sua configuração espacial, fazendo com que o local seja hoje um dos espaços mais importantes da cidade, visto por muitas pessoas como o verdadeiro cartão postal de Presidente Prudente (Silva, 1994).

\section{Métodos}

A metodologia utilizada para a realização deste trabalho está fundamentada na pesquisa bibliográfica e trabalho de campo, utilizando a técnica da observação sistemática através do System for Observing Play and Recreation in Communities (SOPARC).

A metodologia SOPARC decompõe o uso do parque através de uma avaliação momentânea (varreduras ou "scans"), contando o número e tipo de usuários e catalogando suas atividades em um único ponto no tempo. Com múltiplas avaliações, as observações agregadas fornecem uma estimativa do seu uso semanal. Os fatores contextuais foram observados dentro de pré-determinadas "áreas-alvo" relevantes, que foram primeiramente identificadas e mapeadas, para que todas as pessoas pudessem ser observadas e contadas pelos pesquisadores durante a varredura.

A partir disso, os pesquisadores visitaram as áreas-alvo durante os seis primeiros meses do ano de 2015. Realizou-se 4 visitas diárias, em períodos pré-definidos:

- manhã (varredura realizada em horário entre 7h e $10 \mathrm{~h}: 30 \mathrm{~min})$;

- meio do dia (varredura realizada em horário entre 10h:30min e 14h);

- tarde (varredura realizada em horário entre 14h:01min e $17 \mathrm{~h}: 30 \mathrm{~min}$ );

- fim de tarde (varredura realizada em horário entre $17 \mathrm{~h}: 31 \mathrm{~min}$ e $21 \mathrm{~h}$ ).

Foram realizadas 4 observações diárias em 3 dias de cada mês que não estivesse chovendo, sendo 2 dias de semana e 1 dia de fim de semana. Realizou-se, então,
12 observações mensais em cada uma das 32 áreas alvo, sendo 384 observações mensais, e um total de 2.304 durante todo o semestre. As áreas-alvo foram observadas na mesma ordem rotacional durante as visitas, conforme estabelecido pelo manual de utilização do instrumento.

Dois observadores trabalharam juntos para fazer as varreduras. A atividade de cada indivíduo presente no local foi codificada, através do uso de um aplicativo desenvolvido exclusivamente para facilitar o uso do instrumento metodológico e evitar desperdício de papéis - o "iSoparc". Classificou-se então o indivíduo observado como envolvido em atividade sedentária (indivíduo deitado, sentado ou em pé), atividade moderada (indivíduo andando em um ritmo casual), ou atividade vigorosa (indivíduo engajado em uma atividade mais intensa que uma caminhada normal, levando-o a suar e aumentar sua frequência cardíaca, como um trote, corrida, bombeando as pernas em um balanço, etc.).

Além disso, as digitalizações apontavam o sexo (masculino ou feminino) e a idade estimada dos participantes (crianças de 0 a 12 anos, adolescentes de 13 a 20 anos, adultos de 21 a 59 anos e idosos acima de 60 anos). A confiabilidade foi então checada com um terceiro observador e indicou que o processo tinha boa reprodutibilidade, com acordo entre independentes observadores sendo maior do que 0,8 para variáveis relacionadas com a pessoa e superior 0,9 para variáveis relacionadas com a área (Mckenzie et al., 2006).

As áreas-alvo em que as observações se iniciaram foram aquelas com maior fluxo de pessoas, conforme orientado pelo manual de utilização do instrumento. A partir disso, respeitou-se o sentido horário, sendo completada uma quadra de cada vez. Para facilitar a apresentação dos resultados, as 32 áreas-alvo foram agrupadas em 6 quadras, conforme pode ser observado na Figura 3.

Todas as análises estatísticas foram feitas através da utilização de programação específica em linguagem Python e SQL (Structured Query Language). Os dados foram verificados duas vezes pelo investigador principal para a sua validação. A verificação deste também incluiu uma avaliação dos meios, desvios-padrão, frequências e intervalos interquartis para ajudar a identificar possíveis erros na entrada de dados.

As notas de campo, com base em observações sistemáticas foram analisadas tematicamente com 


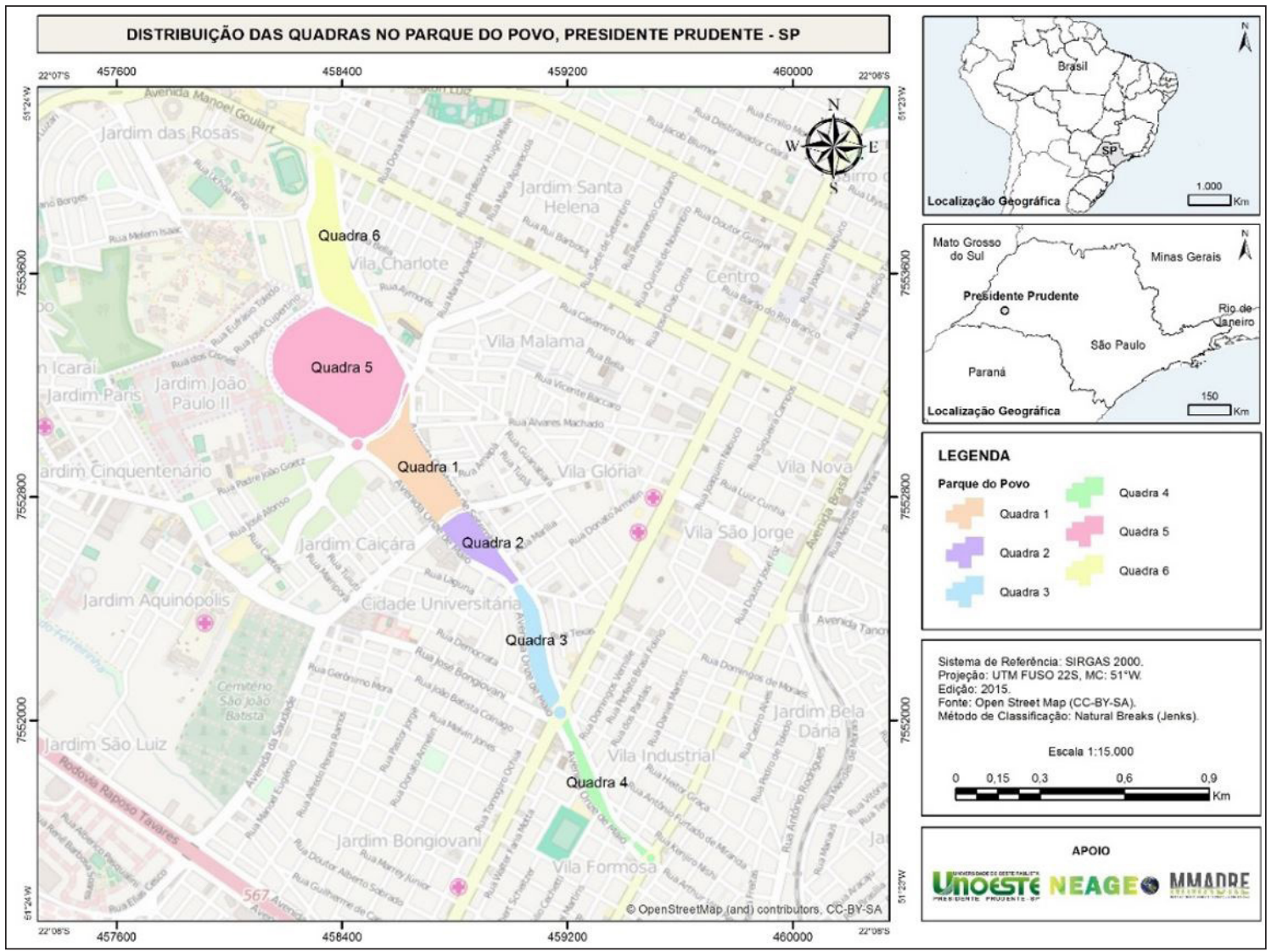

Figura 3 - Distribuiç̧̃o das quadras

Fonte: Trabalho de campo, 2015.

relação às variáveis e objetivos do estudo. As anotações foram então classificadas, e os temas e descobertas observacionais foram posteriormente discutidos, para minimizar o viés do pesquisador na interpretação dos resultados.

\section{Resultados}

Um total de 19.105 indivíduos foi observado durante todo o semestre. 0 período da noite foi o mais frequentado (53\%), seguido do período da tarde (23\%), manhã (16\%) e meio do dia (8\%). Em relação ao nível de atividade encontrado no parque, tanto nos dias de semana como aos finais de semana as atividades moderadas prevalecem (54\%), seguidas das atividades sedentárias (27\%) e por último as atividades vigorosas (19\%) (Tabela 2). As atividades sedentárias aumentam aos finais de semana quando comparadas aos dias de semana $(13 \%$ a mais no público feminino e cerca de $9 \%$ no público masculino).
As atividades moderadas prevaleceram em todos os períodos do dia, e as atividades vigorosas foram mais frequentes no período da noite em ambos os sexos.

Em relação à faixa etária observada no parque, um maior número de adultos (59\%) utilizou o parque que adolescentes (21\%), idosos (13\%) e crianças (7\%).

A Tabela 2 mostra que o número de crianças aumentou aos finais de semana, principalmente as meninas. $\mathrm{O}$ número de população idosa diminuiu consideravelmente aos finais de semana em ambos os sexos, enquanto o fluxo de adolescentes e adultos permaneceu semelhante.

As diferentes faixas etárias estiveram mais presentes em períodos do dia alternados. Os idosos fizeram uso do parque com mais frequência no período da manhã, os adolescentes foram mais presentes no meio do dia, as crianças no período da tarde e os adultos no período da noite (Tabela 3).

Mais homens do que mulheres (homens $=65 \%$, mulheres $=35 \%$ ) utilizaram o parque durante o semestre, e eles estiveram mais envolvidos em atividades vigorosas 
Tabela 2 - Nível de Atividade por dia da semana e por período do dia

\begin{tabular}{|c|c|c|c|c|c|c|}
\hline \multirow{2}{*}{ Período } & \multicolumn{3}{|c|}{ Mulher } & \multicolumn{3}{|c|}{ Homem } \\
\hline & Sedentário & Moderado & Vigoroso & Sedentário & Moderado & Vigoroso \\
\hline \multicolumn{7}{|c|}{ Periodo da Semana } \\
\hline Dia de Semana & $19,25 \%$ & $68,35 \%$ & $12,40 \%$ & $26,21 \%$ & $51,71 \%$ & $22,08 \%$ \\
\hline Final de Semana & $32,64 \%$ & $53,08 \%$ & $14,28 \%$ & $34,71 \%$ & $43,29 \%$ & $22,00 \%$ \\
\hline \multicolumn{7}{|l|}{ Periodo do Dia } \\
\hline Manhã & $5,07 \%$ & $79,87 \%$ & $15,06 \%$ & $9,92 \%$ & $71,68 \%$ & $18,40 \%$ \\
\hline Almoço & $34,85 \%$ & $61,34 \%$ & $3,81 \%$ & $40,94 \%$ & $50,39 \%$ & $8,67 \%$ \\
\hline Tarde & $40,48 \%$ & $53,59 \%$ & $5,94 \%$ & $37,60 \%$ & $40,88 \%$ & $21,52 \%$ \\
\hline Noite & $22,32 \%$ & $61,57 \%$ & $16,11 \%$ & $27,90 \%$ & $46,72 \%$ & $25,38 \%$ \\
\hline
\end{tabular}

Fonte: Trabalho de campo, 2015.

Tabela 3 - Característica do usuário do parque por período da semana/período do dia

\begin{tabular}{|c|c|c|c|c|c|c|c|c|}
\hline \multirow{2}{*}{ Período } & \multicolumn{4}{|c|}{ Mulher } & \multicolumn{4}{|c|}{ Homem } \\
\hline & Criança & Jovem & Adulto & Idoso & Criança & Jovem & Adulto & Idoso \\
\hline \multicolumn{9}{|l|}{ Período da Semana } \\
\hline Dia de Semana & $3,45 \%$ & $15,76 \%$ & $65,74 \%$ & $15,05 \%$ & $7,70 \%$ & $23,73 \%$ & $53,96 \%$ & $14,61 \%$ \\
\hline Final de Semana & $12,82 \%$ & $13,60 \%$ & $66,88 \%$ & $6,70 \%$ & $9,54 \%$ & $25,10 \%$ & $57,63 \%$ & $7,73 \%$ \\
\hline \multicolumn{9}{|l|}{ Período do Dia } \\
\hline Manhã & $0,43 \%$ & $6,52 \%$ & $65,53 \%$ & $27,52 \%$ & $2,95 \%$ & $9,50 \%$ & $56,34 \%$ & $31,21 \%$ \\
\hline Almoço & $6,17 \%$ & $37,02 \%$ & $51,36 \%$ & $5,44 \%$ & $11,08 \%$ & $33,43 \%$ & $47,50 \%$ & $8,00 \%$ \\
\hline Tarde & $11,03 \%$ & $20,66 \%$ & $58,06 \%$ & $10,25 \%$ & $14,65 \%$ & $36,63 \%$ & $41,20 \%$ & $7,52 \%$ \\
\hline Noite & $6,83 \%$ & $13,04 \%$ & $71,13 \%$ & $9,00 \%$ & $6,21 \%$ & $20,55 \%$ & $62,30 \%$ & $10,94 \%$ \\
\hline
\end{tabular}

Fonte: Trabalho de campo, 2015.

do que elas (homens=27\%, mulheres=23\%), além de ocuparem em número desproporcionalmente maior a área com maior número de instalações desportivas. As mulheres, em geral, estiveram mais engajadas em atividades moderadas (63\%) que os homens (46\%).

As quadras 1 e 2 , que possuem um maior número de instalações como pistas de caminhada/corrida, ciclismo e skate, campos de futebol, ginásios e quadras, playgrounds, além de equipamentos de ginástica, foram as mais frequentadas. Observou-se uma preferência dos idosos pela quadra 4, que possui instalações voltadas à caminhada/ corrida, playground e equipamentos de ginástica. As quadras 3 e 5, com menos instalações e mais espaço livre, foram as menos utilizadas no geral.

\section{Discussão}

Este estudo procurou elucidar as características do uso do Parque do Povo na prática de atividades físicas em Presidente Prudente/SP. Adultos e homens foram os grupos observados com maior frequência. Os resultados encontrados demonstraram que o parque é bastante utilizado na busca por saúde na população, já que as atividades moderadas prevaleceram em mais de $50 \%$, o que está de acordo com outros estudos realizados no Brasil (Reis, 2001; Cassou, 2009; Hino et al., 2010), e desacordo com estudos realizados nos Estados Unidos e na Austrália, onde as atividades passivas superaram muito a prática de $\mathrm{AF}$ regular (Giles-Corti et al., 2005; Brownson et al., 2000). 
Apesar de os dados do IBGE trazerem as informações que $46 \%$ da população brasileira é sedentária (IBGE, 2014), os dados coletados podem sugerir que os parques verdes urbanos recebem maior importância na busca de saúde através da AF no Brasil, quando comparados aos outros países estudados. Além disso, o fator econômico influencia a escolha desses locais (Veitch et al., 2012), e o acesso gratuito pode estar relacionado a essa preferência.

A caminhada foi a atividade mais observada no parque, complementando os achados de Malta et al. (2009), que apontam que a caminhada é a AF mais comum praticada nos horários de lazer nas capitais brasileiras, tanto para homens (28\%) como para mulheres (61\%). O Parque do Povo possui calçadas em todo seu arredor, além de pistas para caminhada no interior de suas quadras, em toda a sua extensão.

Apesar de o Censo 2000 relatar que a proporção de homens e mulheres na cidade de Presidente Prudente é praticamente a mesma ( $48 \% \times 52 \%$, respectivamente), os homens estiveram muito mais presentes no parque (65\%) que as mulheres (35\%). Esse fator também foi observado em parques da Europa (Hillsdon et al., 2006; Bedimo-Rung et al., 2005) e Canadá (Cohen et al., 2007), porém não foi observado em estudos realizados no sul do Brasil por Reis (2001), Cassou (2009) e Hino et al. (2010), que encontraram uma divisão de gênero bastante proporcional nas cidades de Curitiba e Florianópolis. Os homens também prevaleceram nos achados de Parra et al. (2010), em estudo realizado no Recife, porém essa diferença foi menos significativa (homens $=56 \%$, mulheres $=44 \%$ ). Além disso, os autores também observaram que nos parques de Pernambuco em que havia programas gratuitos de incentivo à saúde, como aulas de ginástica e aconselhamentos nutricionais, o número de mulheres aumentou significativamente. Esses resultados sugerem que as mulheres de determinadas regiões do país podem estar mais conscientes quanto a necessidade de $\mathrm{AF}$ no dia a dia, e ainda, que a utilização de espaços públicos abertos para a implementação de programas de promoção de saúde é uma estratégia eficiente em prol da saúde da mulher brasileira.

A partir da Figura 4, podemos verificar que os homens estão mais envolvidos em todos os níveis de atividade física, em especial sedentário e vigoroso. 0 fato de os homens estarem mais presentes no parque, se engajarem em mais atividades vigorosas do que as mulheres e ocuparem em número desproporcionalmente maior na área com mais instalações desportivas, pode ser parcialmente explicado através do tipo de atividade escolhida. Evidências mostram que no Brasil, os homens preferem jogar futebol e as mulheres preferem a caminhada como principal AF de lazer (Malta et al., 2009). Atividades como uma partida de futebol pode envolver vários homens ao mesmo tempo, no mesmo local, o que elevaria o número de indivíduos observados em um dado momento.

O baixo número de crianças observado no estudo (7\%) chama a atenção, já que o parque oferece playgrounds em toda a sua extensão, bem como áreas de uso comum que poderiam ser desfrutadas por essa faixa etária. Além disso, o público infantil aumenta aos finais de semana, o que sugere um acordo com os achados de Hino et al. (2010), que constatou que as praças recebem mais crianças que os parques, e que isso se deve ao fator proximidade, já que as praças estariam mais próximas às residências do que os grandes parques, o que acaba por influenciar diretamente na disponibilidade diária dos adultos em acompanhar as crianças para atividades ao ar livre.

A distribuição da população acima de 60 anos também trouxe dados importantes: o período da manhã foi o favorito e sua frequência diminuiu aos finais de semana. Esses achados podem estar atribuídos a peculiaridades próprias da faixa etária, que são adeptos a rotinas e vida diurna. Outro fator interessante é que uma das áreas menos frequentadas pela população em geral foi preferida por essa faixa etária. Nota-se que essa área possui pistas de caminhada em todo seu interior e arredores, fator que vai de encontro aos estudos de Cohen et al. (2007), que também observaram que o parque com um maior número de pistas de caminhada

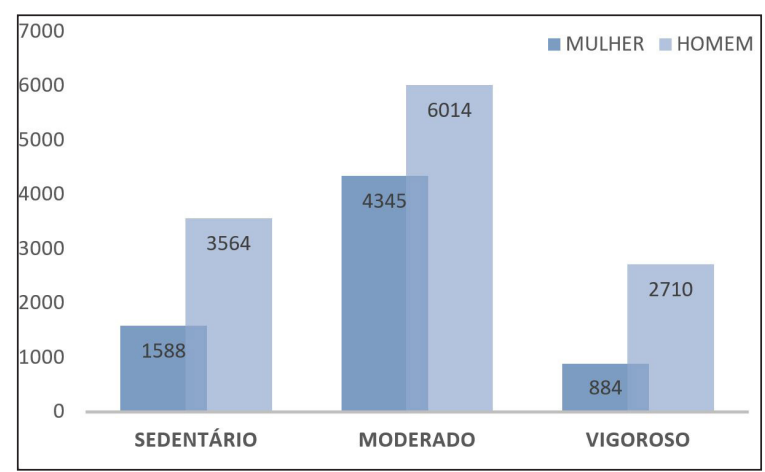

Figura 4 - Quantidade de frequentadores observados no Parque do Povo, distribuídos por nível de atividade física e gênero

Fonte: Trabalho de campo, 2015. 
foi o mais frequentado por idosos. Os autores também sugerem que os idosos precisam de programas e incentivos para ser atraídos aos parques, e nota-se que as "academias da terceira idade" instaladas ao longo do Parque do Povo destacavam-se pela presença deles ao longo das observações.

O nível de AF observado variou muito de acordo com o período do dia. As atividades vigorosas prevaleceram no período da noite (entre $17 \mathrm{~h}: 31 \mathrm{~min}$ e $21 \mathrm{~h}$ ), e as atividades sedentárias foram mais observadas por volta do meio dia. Nesse "horário de almoço" foi observada também uma acentuada diminuição da frequência e do nível de AF dos usuários, o que pode sugerir que a maior parte dos frequentadores do parque sejam trabalhadores que buscam exercitar-se após o horário de serviço, ou até mesmo que o clima mais ameno no fim de tarde é mais atrativo ao público. Um maior número de adolescentes foi observado no meio do dia, fato que pode estar relacionado ao número de escolas na região e aos seus respectivos horários de saída.

A diferença no número de pessoas observados em cada uma das seis quadras do parque é um fator importante a ser analisado. As duas quadras com maior número de instalações e equipamentos disponíveis receberam mais da metade das visitas durante o semestre. Para Cohen et al. (2007) e Silva et al. (2009), os recursos e equipamentos são mais importantes do que o tamanho do parque ou a estética. É possível perceber que esse tipo de facilidade também atrai os frequentadores do Parque do Povo, já que a quadra com maior quantidade de árvores e vegetação foi responsável por menos de $10 \%$ da frequência.

Ademais, a segurança percebida também é um fator observável que influencia o uso de parques urbanos (Bedimo-Rung et al., 2005; Broomhall, 2006), destacando-se o fato de a quadra mais frequentada do parque possuir um posto policial em seu interior. Kaczynski et al. (2008), por sua vez, não perceberam a segurança como fator influente na escolha de parques para a prática de atividades físicas no Canadá. Essa diferença pode se dar ao fato de a segurança ser uma preocupação maior nos países em desenvolvimento.

Outro fator que pode ter influenciado o favoritismo da quadra 1 é o fato de ela possuir uma área maior de estacionamento, o que estaria de acordo com os estudos realizados em Curitiba (Cassou, 2009), que também perceberam o estacionamento como um dos principais responsáveis pela escolha do local.
Autores que fizeram uso do SOPARC no estado do Pernambuco (Parra et al., 2010) constataram que os parques urbanos em que foram implementadas ações governamentais de incentivo à saúde (ginástica, dança, caminhada, corrida, jogos, brincadeiras, palestras, oficinas, reuniões e serviços de orientação nutricional) receberam um aumento significativo do número de usuários, com destaque para o público feminino e idoso, além de obter um importante aumento no número de atividades vigorosas sendo realizadas. Percebe-se que a utilização de espaços públicos para programas como esse podem trazer muito mais do que ganhos em saúde, mas também a minimização de contrastes sociais e econômicos, através da oferta de serviços públicos que garantam a inclusão social e a melhoria da qualidade de vida da população.

Durante as observações, muitas áreas estiveram vazias em dias e períodos diversos. Percebe-se que, considerando todo o tempo que não é utilizado, o local poderia ter um impacto muito maior na população se fosse mais bem aproveitado. Sabe-se que instalações e equipamentos em geral atraem o público (Cohen et al., 2007; Veitch et al., 2012), e que o Parque do Povo tem passado por uma série de reformas para esse fim. Porém, um ganho muito maior poderia vir do agendamento de eventos e atividades organizadas em prol da busca por saúde da população, visto que já existem estudos que comprovam a eficiência desse tipo de ação em espaços urbanos (Parra et al., 2010). Planejadores, supervisores e profissionais de saúde precisariam dispensar especial atenção a esse tipo de programa, no contexto de inversão de prioridades através do investimento na substituição da cultura da medicalização pela cultura de promoção da saúde.

Algumas limitações desse estudo podem ser identificadas. Esse tipo de observação sistemática não elucida as razões que levaram ao comportamento encontrado no parque e outras associações significativas que foram levantadas. Além disso, todas as observações foram realizadas em um mesmo parque, no interior do Estado de São Paulo, e os resultados refletem a realidade ambiental, política, social e cultural do local. A cidade é conhecida por seu clima quente durante a maior parte do ano, o que pode gerar uma grande influência na frequência e na prática de atividades físicas em ambientes abertos durante o dia.

Além disso, este estudo tem vários pontos fortes que devem ser destacados. Apesar de a metodologia SOPARC ser baseada em amostras momentâneas, 
proporcionando apenas uma informação instantânea sobre o ambiente, fora realizada uma série sistemática de observações em busca de aumentar a validade dos resultados. Os observadores foram treinados para alcançar um elevado nível de concordância, fornecendo adequada confiabilidade. Embora o número de estudos que associa o uso de parques com os níveis de $\mathrm{AF}$ esteja aumentando nos últimos anos, esse tipo de pesquisa ainda é escasso no Brasil, e na maioria das vezes concentrados na região sul do país.

\section{Conclusão}

Esse trabalho mostrou uma relação positiva entre áreas verdes urbanas e encorajamento para a prática de atividades físicas. Sabe-se que uma vida fisicamente ativa traz inúmeros benefícios em muitos determinantes de saúde, e que ações voltadas para esse fim devem receber atenção, já que quase metade da população do país pode ser considerada sedentária. A falta de recursos para participar de atividades ou instalações pagas é uma das principais barreiras na busca de atingir os 150 minutos de AF por semana (Hino et al., 2010), recomendados pela Organização Mundial de Saúde. Nesse sentido, a implantação de espaços urbanos de qualidade e melhoria daqueles já existentes, além da implantação de programa de incentivo à prática de atividades físicas nesses locais tem de ser vistos com devida importância na estratégia de promoção de saúde - direito de todos.

Evidências encontradas nesse estudo esclarecem que determinados períodos do dia e da semana, além de características de gênero e faixa etária devem ser considerados para o aumento da eficácia de programas relacionados à promoção da saúde através da AF. Estratégias dirigidas ao público infantil devem receber atenção na região, já que poucas crianças foram observadas, e a probabilidade de uma criança ativa se tornar um adulto também ativo tem sido constantemente relatada em estudos recentes (Lazzoli et al., 1998). Promover a AF na infância e na adolescência pode significar uma base sólida para a redução da prevalência do sedentarismo na idade adulta, e programas com esse objetivo poderiam ser oferecidos aos finais de semana, no período da tarde.

\section{Referências}

Amorim, M. C. C. T. (1993). Análise ambiental e qualidade de vida na cidade de Presidente Prudente/SP (Dissertação de mestrado). Universidade Estadual Paulista "Júlio de Mesquita Filho", Faculdade de Ciências e Tecnologia, Presidente Prudente.

Bargos, D. C. (2010). Mapeamento e análise das áreas verdes urbanas como indicador da qualidade ambiental urbana: estudo de caso de Paulínia-SP (Dissertação de mestrado). Instituto de Geociências, Universidade Estadual de Campinas, Campinas.

Bedimo-Rung, A. L., Mowen, A. J., \& Cohen, D. A. (2005). The significance of parks to physical activity and public health: a conceptual model. American Journal of Preventive Medicine, 28(2, Suppl 2), 159-168. http://dx.doi.org/10.1016/j. amepre.2004.10.024. PMid:15694524.

Branas, C. C., Cheney, R. A., MacDonald, J. M., Tam, V. W., Jackson, T. D., \& Ten Have, T. R. (2011). A difference-indifferences analysis of health, safety, and greening vacant urban space. American Journal of Epidemiology, 174(11), 1296-1306. http://dx.doi.org/10.1093/aje/kwr273. PMid:22079788.

Broomhall, M. H. (2006). Study of the availability and environmental quality of urban open space used for physical activity (Dissertação de mestrado). University of Western Australia, Perth.

Brownson, R. C., Jones, D. A., Pratt, M., Blanton, C., \& Heath, G. W. (2000). Measuring physical activity with the behavioral risk factor surveillance system. Medicine and Science in Sports and Exercise, 32(11), 1913-1918. http://dx.doi.org/10.1097/00005768-200011000-00015. PMid:11079522.

Cassou, A. C. N. (2009). Características ambientais, frequência de utilização e nível de atividade física dos usuários de parques e praças de Curitiba - PR (Dissertação de mestrado). Universidade Federal do Paraná, Curitiba.

Cohen, D. A., McKenzie, T. L., Sehgal, A., Williamson, S., Golinelli, D., \& Lurie, N. (2007). Contribution of public parks to physical activity. American Journal of Public Health, 97(3), 509-514. http://dx.doi.org/10.2105/AJPH.2005.072447. PMid:17267728.

Collet, C., Chiaradia, B. M., Reis, R. S., Nascimento, J. V. (2012).

Fatores determinantes para a realização de atividades físicas 
em parque urbano de Florianópolis. Revista Brasileira de Atividade Física \& Saúde, 13(1), 15-23.

Coombes, E., Jones, A. P., \& Hillsdon, M. (2010). The relationship of physical activity and overweight to objectively measured green space accessibility and use. Social Science \& Medicine, 70(6), 816-822. http://dx.doi. org/10.1016/j.socscimed.2009.11.020. PMid:20060635.

Díaz, G. (2005). Vegetacion y calidad ambiental de las ciudades. Arquitectura y Urbanismo, 26(1), 44-49.

Giles-Corti, B., Broomhall, M. H., Knuiman, M., Collins, C., Douglas, K., Ng, K., Lange, A., \& Donovan, R. J. (2005). Increasing walking: how important is distance to, attractiveness, and size of public open space? American Journal of Preventive Medicine, 28(2, Suppl 2), 169176. http://dx.doi.org/10.1016/j.amepre.2004.10.018. PMid:15694525.

Hillsdon, M., Panter, J., Foster, C., \& Jones, A. (2006). The relationship between access and quality of urban green space with population physical activity. Public Health, 120(12), 1127-1132. http://dx.doi.org/10.1016/j.puhe.2006.10.007. PMid:17067646.

Hino, F.A.A., Reis, R. S., Ribeiro, I. C., Parra, D. C., Brownson, R. C., \& Fermino, R. C. (2010). Using observational methods to evaluate public open spaces and physical activity in Brazil. Journal of Physical Activity \& Health, 7(s2), 146-154. http://dx.doi.org/10.1123/jpah.7.s2.s146. PMid:20702903.

Hoehner, C. M., Brennan Ramirez, L. K., Elliott, M. B., Handy, S. L., \& Brownson, R. C. (2005). Perceived and objective environmental measures and physical activity among urban adults. American Journal of Preventive Medicine, 28(2, Suppl 2), 105-116. http://dx.doi.org/10.1016/j. amepre.2004.10.023. PMid:15694518.

IBGE. (2014). Pesquisa Nacional de Saúde 2013: percepção do estado de saúde, estilos de vida e doenças crônicas - Brasil, grandes regiões e unidades da federação. Rio de Janeiro: IBGE. Recuperado em 10 de janeiro de 2016, de ftp://ftp. ibge.gov.br/PNS/2013/pns2013.pdf.

INMET. (2017). Banco de dados meteorológicos para ensino e pesquisa. Brasília: INMET.

IPMet. (2005). Presidente Prudente - SP: dados climatológicos diários correspondentes ao período de 1976 a 2003. Presidente Prudente: Estação climatológica FCT, Universidade Estadual Paulista "Júlio de Mesquita Filho".
Kaczynski, A. T., \& Henderson, K. A. (2007). Environmental correlates of physical activity: a review of evidence about parks and recreation. Leisure Sciences, 29(4), 315-354. http://dx.doi.org/10.1080/01490400701394865.

Kaczynski, A. T., Potwarka, L. R., \& Saelens, B. E. (2008). Association of park size, distance, and features with physical activity in neighborhood parks. American Journal of Public Health, 98(8), 1451-1456. http://dx.doi.org/10.2105/ AJPH.2007.129064. PMid:18556600.

Lazzoli, J. K., Nóbrega, A. C. L., Carvalho, T., Oliveira, M. A. B., Teixeira, J. A. C., Leitão, M. B., Leite, N., Meyer, F., Drummond, F. A., Pessoa, M. S. V., Rezende, L., Rose, E. H., Barbosa, S. T., Magni, J. R. T., Nahas, R. M., Michels, G., \& Matsudo, V. (1998). Atividade física e saúde na infância e adolescência. Revista Brasileira de Medicina do Esporte, 4(4), 107-109. http://dx.doi.org/10.1590/S1517-86921998000400002.

Maas, J., Verheij, R. A., Spreeuwenberg, P., \& Groenewegen, P. P. (2008). Physical activity as a possible mechanism behind the relationship between green space and health: a multilevel analysis. BMC Public Health, 8, 206-212. http:// dx.doi.org/10.1186/1471-2458-8-206. PMid:18544169.

Malta, D. C., Moura, E. C., Castro, A. M., Cruz, D. K. A., Morais Neto, O. L., \& Monteiro, C. A. (2009). Padrão de atividade física em adultos brasileiros: resultados de um inquérito por entrevistas telefônicas, 2006. Epidemiologia e Serviços de Saúde : Revista do Sistema Unico de Saúde do Brasil, 18(1), 7-16. http://dx.doi.org/10.5123/S1679-49742009000100002.

Mckenzie, T. L., Cohen, D. A., Sehgal, A., Williamson, S., \& Golinelli, D. (2006). System for Observing Play and Recreation in Communities (SOPARC): reliability and feasibility measures. Journal of Physical Activity \& Health, 3(s1), S208-S222. http://dx.doi.org/10.1123/jpah.3.s1.s208.

Nunes, M. (1999). Os grandes desafios da autarquia no âmbito do desporto, uma proposta de elaboração de um plano de desenvolvimento desportivo municipal. Revista Horizonte, 15(89), 33-39.

Ord, K., Mitchell, R., \& Pearce, J. (2013). Is level of neighbourhood green space associated with physical activity in green space? The International Journal of Behavioral Nutrition and Physical Activity, 10(1), 127-134. http:// dx.doi.org/10.1186/1479-5868-10-127. PMid:24219824.

Owen, N., Humpel, N., Leslie, E., Bauman, A., \& Sallis, J. F. (2004). Understanding environmental influences on walking: review and research agenda. American Journal of Preventive 
Medicine, 27(1), 67-76. http://dx.doi.org/10.1016/j. amepre.2004.03.006. PMid:15212778.

Parra, D. C., McKenzie, T. L., Ribeiro, I. C., Ferreira Hino, A. A., Dreisinger, M., Coniglio, K., Munk, M., Brownson, R. C., Pratt, M., Hoehner, C. M., \& Simoes, E. J. (2010). Assessing physical activity in public parks in Brazil using systematic observation. American Journal of Public Health, 100(8), 1420-1426. http://dx.doi.org/10.2105/AJPH.2009.181230. PMid:20558792.

Presidente Prudente. Prefeitura municipal.(2014). Recuperado em 27 de janeiro de 2016, de http://www. presidenteprudente.sp.gov.br

Reis, R. S. (2001). Determinantes ambientais para a realização de atividades físicas nos parques urbanos de Curitiba: uma abordagem sócio-ecológica da percepção dos usuários (Dissertação de mestrado). Centro de Desportos, Universidade Federal de Santa Catarina, Florianópolis.

Reis, R. S., Hino, A. A., Florindo, A. A., Añez, C. R., \& Domingues, M. R. (2009). Association between physical activity in parks and perceived environment: a study with adolescents. Journal of Physical Activity \& Health, 6(4), 503-509. http://dx.doi.org/10.1123/jpah.6.4.503. PMid:19842465.

Silva, D. A. S., Petroski, E. L., \& Reis, R. S. (2009). Barreiras e facilitadores de atividades físicas em freqüentadores de parques públicos. Motriz, 15(2), 219-227.

Silva, J. A. (2001). Recursos de apoio didático-pedagógico na educação ambiental (Dissertação de mestrado). Faculdade de Ciências e Tecnologia, Universidade Estadual Paulista “Júlio de Mesquita Filho”, Presidente Prudente.

Silva, M. J. M. (1994). O parque do povo em Presidente Prudente - SP: a lógica da intervenção do poder público local no processo de (re)estruturação do espaço urbano
(Dissertação de mestrado). Faculdade de Ciências e Tecnologia, Universidade Estadual Paulista "Júlio de Mesquita Filho", Presidente Prudente.

Sugiyama, T., Francis, J., Middleton, N. J., Owen, N., \& GilesCorti, B. (2010). Associations between recreational walking and attractiveness, size, and proximity of neighborhood open spaces. American Journal of Public Health, 100(9), 1752-1757.http://dx.doi.org/10.2105/AJPH.2009.182006. PMid:20634455.

Tinsley, H. E. A., Tinsley, D. J., \& Croskeys, C. E. (2002). Park usage, social milieu, and psychosocial benefits of park use reported by older urban park users from four ethnic groups. Leisure Sciences, 24(2), 199-218. http:// dx.doi.org/10.1080/01490400252900158.

Veitch, J., Ball, K., Crawford, D., Abbott, G. R., \& Salmon, J. (2012). Park improvements and park activity: a natural experiment. American Journal of Preventive Medicine, 42(6), 616-619. http://dx.doi.org/10.1016/j.amepre.2012.02.015. PMid:22608379.

Veitch, J., Carver, A., Hume, C., Crawford, D., Timperio, A., Ball, K., \& Salmon, J. (2014). Are independent mobility and territorial range associated with park visitation among youth? The International Journal of Behavioral Nutrition and Physical Activity, 11(1), 73-82. http://dx.doi. org/10.1186/1479-5868-11-73. PMid:24909862.

Witten, K., Hiscock, R., Pearce, J., \& Blakely, T. (2008). Neighbourhood access to open spaces and the physical activity of residents: a national study. Preventive Medicine, 47(3), 299-303. http://dx.doi.org/10.1016/j.ypmed.2008.04.010 PMid:18533242.

Recebido em: Out. 13, 2017

Aprovado em: Mar. 19, 2018 